\title{
Invited letter to the editor on the editorials on "High dose irradiation after pleurectomy/decortication or biopsy for pleural mesothelioma treatment"
}

\author{
Elisabetta Parisi, Antonino Romeo \\ Radiotherapy Unit, Istituto Scientifico Romagnolo per lo Studio e la Cura dei Tumori (IRST) IRCCS, Meldola, Italy \\ Correspondence to: Elisabetta Parisi, MD. Istituto Scientifico Romagnolo per lo Studio e la Cura dei Tumori (IRST) IRCCS, via P. Maroncelli 40, \\ 47014 Meldola, Italy. Email: elisabetta.parisi@irst.emr.it. \\ Provenance: This is an invited article commissioned by the Section Editor Laura Chiara Guglielmetti (Cantonal Hospital Winterthur, Kantonsspital \\ Winterthur, Winterthur, Switzerland). \\ Response to: de Perrot M, Cho J. Non-ablative hypofractionated hemithoracic radiation—a new standard of care in mesothelioma? J Thorac Dis \\ 2018;10:S4088-92. \\ Botticella A, De Ruysscher D. Lung-sparing intensity-modulated radiotherapy in malignant pleural mesothelioma: palliative or potentially radical? J \\ Thorac Dis 2018;10:S4038-9.
}

Submitted Nov 05, 2018. Accepted for publication Nov 09, 2018.

doi: $10.21037 /$ jtd.2018.11.57

View this article at: http://dx.doi.org/10.21037/jtd.2018.11.57

It is with great interest that we read the two editorials, "Non-ablative hypofractionated hemithoracic radiationa new standard of care in mesothelioma?" by de Perrot and Cho (1) and "Lung-sparing intensity-modulated radiotherapy in malignant pleural mesothelioma: palliative or potentially radical?" by Botticella and De Ruysscher (2), both addressed to our previous publication on hypofractionation and pleural mesothelioma (3). We thank the physicians for their comments and take the opportunity of responding in this Letter to the Editor.

Malignant pleural mesothelioma (MPM) is a relatively rare malignancy with a poor prognosis, the median survival after diagnosis being $<12$ months (4). Five-year survival rates of $>20 \%$ have been reported in studies focusing on trimodality treatment with extrapleural-pneumonectomy (EPP) (5). The advent of a new surgical approach, pleurectomy/decortication (P/D), has created a new scenario in the adjuvant setting for radiation oncologists. A way of irradiating all pleural volume, sparing intact lung, has become a compelling challenge. Recent technological advances (6) in radiotherapy have brought about a substantial reduction in lung toxicity, leading to good results for the trimodality approach (7). In our previously published retrospective study (3), we wanted to underline that hypofractionation is technically and clinically possible in patients submitted to $\mathrm{P} / \mathrm{D}$ or only biopsy, despite the amplitude of irradiation volumes. As stated by Botticella and De Ruysscher (2), we are well aware that the first important limitation of our study was its retrospective design, but we do not deny the possibility of standard treatment, when indicated, to anyone. Forty-one percent of patients with stage disease I-II were treated with hypofractionated radiotherapy but also surgery-specifically, 50\% underwent simple pleurectomy, $25 \%$ extended pleurectomy and $25 \%$ partial pleurectomy, which means that they all had residual disease. Literature data (8) underline that macroscopic residual disease should generally be considered a poor prognostic factor. Given the above surgical data, the poor prognosis, the volume of irradiation excluding pleural fissures and the biologically delivered dose, the aim of our treatment became palliative, i.e., to shrink the tumor and slow down its growth. We agree with Botticella and De Ruysscher's suggestion about including these patients in a prospective clinical trial. We have, in fact, designed and activated a prospective phase II trial for MPM patients after chemotherapy and P/D or biopsy using a different treatment schedule: $30 \mathrm{~Gy} / 5$ daily fractions (at the reference isodose of $60-70 \%$ ) with an internal inhomogeneous dose of up to 40 Gy (IRST 163.01). The promising results from our previous retrospective study in which a median overall 
survival (OS) of 21.6 months was obtained encouraged us to design a new protocol aimed at improving progressionfree survival (PFS) and OS without, however, increasing acute and late toxicity. Indeed, the primary objective of the study is the evaluation of toxicity. To date the best results for OS have been obtained by de Perrot et al. in their study of MPM patients undergoing preoperative short-course radiotherapy followed a week later by EPP (9). The authors reported a median OS of 36 months and an ipsilateral pleural recurrence rate of around $13 \%$.

The role of hypofractionation in MPM has recently come under the scrutiny of researchers. In poor prognosis patients, such as those with MPM, we believe that offering radiation treatment of short duration, i.e., 1 week rather than the standard 6 weeks, improves patient compliance and reduces overall treatment costs. Thus, if it were possible to obtain the same OS and PFS rates for conventional fractionation, with similar or lower toxicity, why not administer hypofractionation in an adjuvant setting? Other issues to clarify are the optimal radiotherapy dosage and relative fractionation. de Perrot and Cho (1) correctly defined non-ablative hypofractionation as a dose of $25 \mathrm{~Gy}$ in 5 daily fractions. Surely an ablative hypofractionation with a dose/fraction $\geq 10$ Gy would be too toxic for the entire pleural volume? This means that a non-ablative dose $>5 \mathrm{~Gy} /$ fraction may be adequate to treat pleural mesothelioma, obtaining balanced efficacy and toxicity. All these aspects need to be studied in prospective clinical trials. As de Perrot and Cho suggest in their editorial (1), “... the combined approach of radiotherapy and immunotherapy, within the trimodality treatment of MPM, could lead to important and interesting results." The greatest difficulty would be in monitoring lung toxicity related to the combination of immunotherapy and lung-sparing radiotherapy.

To clarify the situation, combination trials are needed on radiotherapy and targeted immunotherapy with a lower lung toxicity profile and an adequate interval between the two therapies. The rationale for this combination lies in the activation of the immune system by hypofractionation. In the past, the only "space" for radiotherapy in MPM patients was in a palliative setting, but the approach to the disease has completely changed over the past few years thanks to conservative surgical approaches, advances in radiotherapy techniques and equipment, and innovative studies encompassing clinical and preclinical research.

In conclusion, conventional fractionation is currently the only therapeutic indication for adjuvant MPM, and hypofractionation is not contemplated as a standard of care in this patient setting. It can only be administered in prospective clinical trials using intensity-modulated therapy or image-guide radiotherapy, or in a palliative setting, and it is imperative to entrust treatment to centers of excellence in the field.

\section{Acknowledgements}

The authors would like to thank Gráinne Tierney for editorial assistance.

\section{Footnote}

Conflicts of Interest: The authors have no conflicts of interest to declare.

\section{References}

1. de Perrot M, Cho J. Non-ablative hypofractionated hemithoracic radiation - a new standard of care in mesothelioma? J Thorac Dis 2018;10:S4088-92.

2. Botticella A, De Ruysscher D. Lung-sparing intensitymodulated radiotherapy in malignant pleural mesothelioma: palliative or potentially radical? J Thorac Dis 2018;10:S4038-9.

3. Parisi E, Romeo A, Sarnelli A, et al. High dose irradiation after pleurectomy/decortication or biopsy for pleural mesothelioma treatment. Cancer Radiother 2017;21:766-73.

4. Muers MF, Stephens RJ, Fisher P, et al. Active symptom control with or without chemotherapy in the treatment of patients with malignant pleural mesothelioma (MS01): a multicentre randomised trial. Lancet 2008;371:1685-94.

5. de Perrot M, Feld R, Cho BC, et al. Trimodality therapy with induction chemotherapy followed by extrapleural pneumonectomy and adjuvant high-dose hemithoracic radiation for malignant pleural mesothelioma. J Clin Oncol 2009;27:1413-8.

6. Sterzing F, Sroka-Perez G, Schubert K, et al. Evaluating target coverage and normal tissue sparing in the adjuvant radiotherapy of malignant pleural mesothelioma: helical tomotherapy compared with step-and-shoot IMRT. Radiother Oncol 2008;86:251-7.

7. Rosenzweig KE, Zauderer MG, Laser B, et al. Pleural intensity-modulated radiotherapy for malignant pleural mesothelioma. Int J Radiat Oncol Biol Phys 2012;83:1278-83.

8. Hermanek P, Wittekind C. Residual tumor (R) 
classification and prognosis. Semin Surg Oncol 1994;10:12-20.

9. de Perrot M, Feld R, Leighl NB, et al. Accelerated hemithoracic radiation followed by extrapleural pneumonectomy for malignant pleural mesothelioma. J Thorac Cardiovasc Surg 2016;151:468-73.

Cite this article as: Parisi E, Romeo A. Invited letter to the editor on the editorials on "High dose irradiation after pleurectomy/decortication or biopsy for pleural mesothelioma treatment". J Thorac Dis 2018;10(12):E819-E821. doi: 10.21037/ jtd.2018.11.57 